\title{
COMPUTATION OF ELECTRIC DIPOLE MATRIX ELEMENTS FOR HYDROGEN FLUORIDE*
}

\author{
Robert E. Meredith ${ }^{\dagger}$ and Frederick G. Smith ${ }^{\dagger}$ \\ Willow Run Laboratories, Institute of Science and Technology. The University of Michigan. \\ Ann Arbor 48103, U.S.A.
}

(Received 14 March 1972)

\begin{abstract}
The electric dipole matrix elements of hydrogen fluoride have been calculated by numerical integration for transitions involving large quantum numbers $t, J$. Overtones have been included through $\Delta r=5$. Molecular wave functions obtained by numerical integration of the Schrödinger equation were used. The influence of the mechanical motion on the matrix elements has been determined for Morse and Rydberg Klein Rees (RKR) potential functions. The influence of the electric dipole-moment function approximations has been investigated by a comparison of matrix elements obtained with approximations having the form of a truncated polynomial and a wave-function expansion. The inaccuracies in the matrix elements caused hy uncertainties in the dipolemoment coefficients have been investigated
\end{abstract}

\section{INTRODUCTION}

THE ProBabiLITY per sec, $A(u \rightarrow l)$, that a molecule will spontaneously emit radiation and arrive at a lower energy state is a quantity of great importance to many applied physics and engineering problems. The measurement or calculation of $A(u \rightarrow l)$ is exceedingly difficult for most transitions of interest. If appropriate measurements are not available for a particular energy-level system, one must calculate the $A(u \rightarrow l)$ from first principles. If experimental information is available for a given system, it may be possible to model the electrical and mechanical motion of the molecule and, within the limits imposed by the model, $A(u \rightarrow l)$ may be calculated for otherwise inaccessible transitions. ${ }^{(1-3)}$

A case in point is the probability for spontaneous, infrared emission from upper vibration-rotation levels in diatomic molecules. To date, no direct measurements of these probabilities have been made. However, for most molecules, probabilities are known from absolute absorption-strength measurements on transitions involving the lowest vibration-rotation levels. The proportionality between line strength and transition probability is :(4)

$$
S \propto|\langle u|\boldsymbol{\mu}| l\rangle|^{2}
$$

The quantity $\langle u|\boldsymbol{\mu}| l\rangle$ is defined as

$$
\langle u|\boldsymbol{\mu}| l\rangle=\int_{\eta V} \Psi_{u} \mu \Psi_{l} \mathrm{~d} \eta \mathrm{d} V
$$

* This research was sponsored by the Advanced Research Projects Agency under ARPA Order 236, Contract DAHC 15-67-C-0062, and ARPA Order 1180-72, Contract DAAH-01-72-C-0573.

† Present address: Science Applications Incorporated, 5 Research Drive, Ann Arbor, Michigan 48103, U.S.A. 
where the $\Psi$ are solutions of the Schrödinger equation for a system of three nuclear degere of freedom $(V-r, \theta, \phi)$ and $n$ electronic degrees of freedom with coordinates, 1 . The and $l$ are sets of quantum numbers specilying upper and lower stationary energy state of the diatomic molecule. The electric dipole-moment function is a function of $\mu, \% .1 \%$ Extrapolation of $\langle\||\mu| l\rangle$ from small $(u, l)$ values to large values involves: 11$)$ modeling the function $\mu$ : (2) solving a simplified form of the Schrödinger equation : and. (3) performing the integration in equation (1). This extrapolation is the subject of this paper. The remainder of Section 1 reviews concepts basic to an understanding of the model in be developed and a brief review of pertinent literature.

\subsection{Definition of the dipole moment $\mu(r)$}

The basic assumption in molecular theory is the Born (Oppenheimer approximation. ${ }^{15}$ a theory in which the electron motions are assumed to be independent of the vibrational and rotational motion. To this approximation, the solution to the Schrödinger equation is:

$$
\Psi\left(\eta, r,(\eta, \phi)=U_{n}(\eta) \psi(r) Y_{. l}^{M}(t), \phi\right)
$$

where $J$ and $M$ are quantum numbers speeifying the rotational angular momentum and its projection on at space-fixed axis: $U_{n_{j}}$ is the wave function which describes the electronic state with quantum numbers $n_{j} ; Y_{J}^{M}(0, \phi)$ are associated Legendre polynomials: and $\psi(r)$ is a solution of the radial Schrödinger equation:

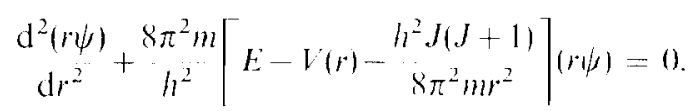

In the above. $h$ is Planck's constant: $m$ is the reduced mass: $E$ is the stationary energy value; and $V(r)$ is the mechanical potential which describes the nuclear motion.

Symmetry requires that in the case of a diatomic molecule. the molecule-fixed dipolemoment function, $\boldsymbol{\mu}_{F}$, be directed along the internuclear axis:

$$
\boldsymbol{\mu}_{F}=\sum_{x} i_{x} q_{x}
$$

where the sum is taken over all electrons and nuclei : $s$ is an effective charge for each particle: and $q_{x}$ is the coordinate along the nuclear axis within the frame of reference fixed in the molecule. The dipole moment $\boldsymbol{\mu}(\eta, r, \theta), \phi)$ is given as a function of direction cosines relative to the laboratory frame $(x, y, z)$ :

$$
\begin{aligned}
& \left.\mu_{x}(\eta) . r, \theta, \phi\right)=\left|\boldsymbol{\mu}_{F}\right| \sin \theta \cos \phi \\
& \left.\mu_{y}(\eta) . r . \theta, \phi\right)=\left|\boldsymbol{\mu}_{F}\right| \sin \theta \sin \phi \\
& \mu_{z}(\eta, r . \theta, \phi)=\left|\boldsymbol{\mu}_{F}\right| \cos \theta .
\end{aligned}
$$

For fixed electronic states, the mean value of $\left|\boldsymbol{\mu}_{F}\right|$ depends strongly on $r$ but not on $\psi$. Therefore. for fixed $n_{j}$. an average electric dipols-moment function may be defined as

$$
\mu(r)=\int U_{n_{j}}^{*} \boldsymbol{\mu}_{F^{\prime}}(\eta, r) L_{n_{j}} \mathrm{~d} \eta
$$


The components of equation (1) may then be rewritten as :

$$
\begin{aligned}
& \left\langle n_{j} l^{\prime} J^{\prime} M^{\prime}\left|\mu_{x}(\eta, r, \theta, \phi)\right| n_{j} \varepsilon J M\right\rangle \\
& \quad=\int_{0}^{\infty} \mathrm{d} r r^{2} \psi_{v^{\prime} J}^{*} \int_{\eta} U_{n_{j}}^{*}\left|\mu_{F}(\eta, r)\right| U_{n_{j}} \mathrm{~d} \eta \psi_{v} J \int_{0}^{\pi} \int_{0}^{2 \pi} \mathrm{d} \theta \sin \theta \mathrm{d} \phi \sin \theta \cos \phi Y_{J^{\prime}}^{M^{*}} Y_{J}^{M}
\end{aligned}
$$

or, if $n_{j}$ is suppressed. as is customary for $n_{j}^{\prime}=n_{j}$

$$
\left\langle r^{\prime} J^{\prime} M^{\prime}\left|\mu_{x}(r)\right| r J M\right\rangle=\int_{0}^{x} \mathrm{~d} r r^{2} \psi_{\nu^{\prime},}^{*}, \mu(r) \psi_{\mathrm{r} J} \int_{0}^{\pi} \int_{0}^{2 \pi} \mathrm{d} \theta \sin \theta \mathrm{d} \phi \sin \theta \cos \phi Y_{J^{\prime}}^{M^{\prime}} Y_{J}^{M}
$$

The procedure is the same for $\mu_{x}$ and $\mu_{z}$. Squaring and summing these results give:

$$
\begin{aligned}
& \left|\left\langle v^{\prime} J^{\prime}|\mu(\eta, r, \theta, \phi)| v J\right\rangle\right|^{2}=\sum_{M^{\prime}, M} \sum_{i=x, y, z}\left|\left\langle v^{\prime} J^{\prime} M^{\prime}\left|\mu_{i}(r, \theta, \phi)\right| v, J, M\right\rangle\right|^{2} \\
& =\sum_{M . M M^{\prime}}\left[\int_{0}^{x} r^{2} \mathrm{~d} r \psi_{I^{\prime}, J}^{*}, \mu(r) \psi_{\cdot J}\right]^{2}\left[\left(\int_{0}^{\pi} \int_{0}^{2 \pi} \mathrm{d} \theta \sin \theta \mathrm{d} \phi Y_{J^{\prime}}^{M^{*}} \sin \theta \cos \phi Y_{I}^{M}\right)^{2}\right. \\
& \left.+\left(\int_{0}^{\pi} \int_{0}^{2 \pi} \mathrm{d} \theta \sin \theta \mathrm{d} \phi Y_{J}^{M^{\prime}} \sin \theta \sin \phi Y_{J}^{M}\right)^{2}+\left(\int_{0}^{\pi} \int_{0}^{2 \pi} \mathrm{d} \theta \sin \theta \mathrm{d} \phi Y_{J^{\prime}}^{M^{*}} \cos \theta Y_{J}^{M}\right)^{2}\right] \\
& \left|\left\langle v^{\prime} J^{\prime}|\mu(r)| r J\right\rangle\right|^{2}=|m|\left[\int_{0}^{r^{2}} r^{2} \mathrm{~d} r \psi_{r^{\prime} J}^{*} \mu(r) \psi \psi_{r J}\right]^{2}
\end{aligned}
$$

where

$$
m= \begin{cases}J+1 & R \text { Branch } \\ -J & P \text { Branch }\end{cases}
$$

The reduction of (9) to (10) is straightforward, and it shows that the factor $|m|$ in (10) arises from the transformation properties of the electric dipole moment. ${ }^{(6)}$ Since $|m|$ is uniquely determined by the quantum number $J$, we will consider only the radial dipole quantities $\left\langle\iota^{\prime} J^{\prime}|\mu(r)| \imath J\right\rangle$.

\subsection{Properties of the $\left\langle v^{\prime} J^{\prime}|\mu(r)| v J\right\rangle$}

The $\left\langle l^{\prime} J^{\prime}|\mu(r)| r J\right\rangle$ form an array, with the rows labeled according to the upper (primed) level involved in a transition, and with the columns labeled according to the lower (unprimed) levels. It can be shown that this array has all the properties of a matrix; consequently, the individual quantities $\left\langle v^{\prime} J^{\prime}|\mu(r)| v J\right\rangle$ are called the matrix elements of the dipole moment. Once they are calculated, the vibrational section rules may be determined for a particular $\mu(r)$ model and potential function. For example consider the lowest order approximation. If it is assumed that $\mu(r)$ is proportional to the displacement from nuclear 
cquilibrium. $r=r-r_{i,}$ then

$$
\mu(x), x
$$

Il it is also assumed that the mechanical motion is harmonic and that there is no interaction between the vibrational and rotational motions, then

$$
\begin{gathered}
J(J+1) \\
r^{2}
\end{gathered}
$$

and

$$
V(x)+x^{2}
$$

In this case, the solutions to (3) are the Hermite polynomials, "and the vibrational matrix elements are independent of $J$ :

$$
\begin{aligned}
& \langle x+1|x| r\rangle=\sqrt{\left(\begin{array}{c}
r+1 \\
2
\end{array}\right)}
\end{aligned}
$$

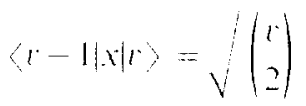

where the rows are labeled by $r^{\prime}=0,1.2 \ldots$ That is:

$\begin{array}{cccc}0 & 12 & 0 & 0 \\ 12 & 0 & 1 & 0 \\ 0 & 1 & 0 & 32 \\ 0 & 0 & 132 & 0\end{array}$

The matrix indicates that in the harmonic approximations (8) $(10)$, only the fundamental series $(\Delta r= \pm 1)$ occurs, and this matrix indicates the relative series values. If more realistic functions of $\mu(r)$ and $V(r)$ are chosen. the zero elements become finite and one predicts overtone series, where $\Delta t>+1$.

Once the electrical and mechanical motion has been modeled and the matrix elements have been determined analytically or numerically, the isotropic transition probabilities (in intensity units) may be found from the following relations: ${ }^{(1)}$

$$
\begin{aligned}
& A\left(r^{\prime} J^{\prime} \rightarrow r J\right)=\frac{64 \pi^{4} y^{3}|m|}{3 h\left(2 J^{\prime}+1\right)}\left|\left\langle v^{\prime} J^{\prime}|\mu(r)| \imath J\right\rangle\right|^{2} \text { (molecule-sec) } \quad 1 \\
& B\left(r^{\prime} J^{\prime} \rightarrow \imath J\right)=\frac{32 \pi^{+}|m|}{3 h^{2} \cdot\left(2 . J^{\prime}+1\right)^{\prime}}\left|\left\langle r^{\prime} J^{\prime}|\mu(r)| r J\right\rangle\right|^{2}\left(\begin{array}{c}
\mathrm{cm}^{2} \\
\text { molecule-erg-sec }
\end{array}\right) \\
& B\left(\imath^{\prime} J^{\prime} \leftarrow \imath J\right)=\frac{\left(2 J^{\prime}+1\right)}{(2 J+1)} B\left(v^{\prime} J^{\prime} \rightarrow \imath J\right) .
\end{aligned}
$$




\subsection{Recent developments in the determination of $\left\langle v^{\prime} J^{\prime}|\mu(r)| v J\right\rangle$}

Integration of equation (1) within the framework of the Born-Oppenheimer approximation has been achieved by several authors using mechanical and electrical models which have varying degrees of sophistication. OPPENHEIMER ${ }^{(8)}$ considered the effects of vibrationrotation interaction $\left(r \neq r_{e}\right.$ in equation 13$)$ on the strength of vibration-rotation lines for harmonic oscillations of the nuclei (equation 14) and for the linear approximation of $\mu(r)$ (equation 12). He found that rotational effects altered the square of the matrix element of each line from the harmonic approximation of equal strength lines, by a correction factor. $F$ :

$$
\left|\left\langle v^{\prime} J^{\prime}\left|\mu\left(r^{\prime}\right)\right| v^{\prime} J\right\rangle\right|^{2}=\left|\left\langle v^{\prime}|\mu(r)| v\right\rangle\right|^{2} F
$$

where $\left\langle v^{\prime}|\mu(r)| v\right\rangle$ is the rotationless matrix element. In the above, for fundamental band $P$ and $R$ branch lines:

$$
\begin{array}{ll}
F=1+4 \gamma J\left[1+\frac{5}{8} \gamma J-\frac{3}{8} \gamma\right] & P \text { Branch } \\
F=1-4 \gamma(J+1)\left[1-\frac{5}{8} \gamma(J+1)-\frac{3}{8} \gamma\right] & R \text { Branch }
\end{array}
$$

where $\gamma=\left(2 B_{e} / \omega_{e}\right)$. Herman and WaLlis ${ }^{(13)}$ extended the Oppenheimer result to include the effects of an anharmonic potential for a dipole-moment function in the form:

$$
\mu(r) \simeq M_{0}+M_{1}\left(r-r_{e}\right) .
$$

The $F$-factors of Herman and Wallis contain a parameter $\theta=M_{0} /\left(M_{1} r_{e}\right)$, which predicts an increase in intensity of the $P$ branch lines over the $R$ branch lines (or vice versa, depending on the sign of $\theta$ ). This model, represented by equation (20), was recently extended to include quadratic and cubic terms in the dipole-moment function. The case of a rotating Morse oscillator was treated by HeAPS and HERZBERG ${ }^{(10)}$ in 1952 and later by Herman et al..$^{(12,13)}$ An alternate analytic approach was taken by TRISCHKA and SALWEN ${ }^{(14)}$ who expressed $\mu(r)$ as a linear expansion of molecular wave functions. This is possible. since the wave functions form a complete orthonormal basis.

More currently, numerical techniques have been used for determination of electric dipole-moment functions and molecular wave functions. ${ }^{(15)} \mathrm{CASHION}^{(16)}$ has tested the validity of empirical potential functions by numerically integrating the Schrödinger equation.

This paper is concerned with the determination of electric dipole matrix elements $\left\langle l^{\prime} J^{\prime}|\mu(r)| u J\right\rangle$ by numerical integration of the Schrödinger equation and the $r$-dependent integral which appears in equation (10). The influence of $V(r)$ on the vibrational matrix elements will be investigated by a numerical integration of equation (3) for $J=0$, for several different functions $V(r)$. The influence of $V(r)$ and the vibration-rotation interaction will be determined by repetition of these computations for $J>0$. The influence of the form of $\mu(r)$ on the matrix elements will be investigated by numerical integration of equation (10) for the polynomial form of $\mu(r)$ :

$$
\mu_{p}(r)=\sum_{i} M_{i}\left(r-r_{e}\right)^{i} \cong M_{0}+M_{1}\left(r-r_{e}\right)+\cdots
$$

and for the wave-function expansion of Trischka and Salwen:

$$
\mu_{w}(r)=\sum_{i} A_{i} \frac{\psi_{i}(r)}{\psi_{0}(r)}=A_{0}+A_{1} \frac{\psi_{1}(r)}{\psi_{0}(r)}+\cdots .
$$


Application of these computations will be made to the high $r, J$ transitions of the IIt molecule. for the pure rotation. fundamental, and overtone bands. The rotationless wave functions $\psi_{*}$ will be used for $\mu_{n}$ even for the $J$ dependent matrix elements. a procedure analogous to that used with the polynomial expansion.

\section{INFLLENCE OF I $(r)$ ON THF MATRIX ELEMENTS}

\subsection{Morse potential}

A number of potential functions have been used in the calculation of dipole-moment matrix elements. Because a comprehensive evaluation of the various forms of potentials has been given in the literature. ${ }^{(1.18)}$ an extensive comparison will not be attempted here. Rather, we have chosen to compare results obtained from the Morse potential, one of the most simple and commonly used empirical potentials, wh the Rydberg Klein Rees potential (RKR). a form which gives better agreement with the true energy levels of the molecule.

The expression which we have used for the Morse potential is

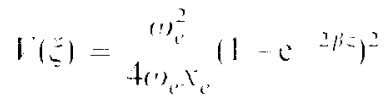

where

$$
\beta=\left(\begin{array}{l}
(1)_{1} x_{0} \\
4 B_{1}
\end{array}\right)^{1 \cdot 2}
$$

and

$$
\zeta=r-r
$$

This type of empirical potential function was originally used becaluse it allows a closedform solution to the radial Schrodinger equation and it reproduces the energy levels of most molecules reasonably well. More important here, this potential is casily constructed with just three independent parameters. which are defined in terms of spectroscopic constants known for virtually all diatomic molecules. Thus. if this potential yields good results for matrix-element calculations for $\mathrm{HF}$ tone of the more difficult molecules (o) model because of its high degree of anharmonicity). then it should yield reasonable results for most diatomic molecules.

\subsection{RKR potential}

The RKR potential function is obtained by a Wentzel Kramers Brillouin (WKB)

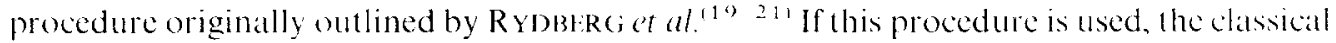
lurning points of the vibrational motion are determined directly from the observed encrgy level transitions of a particular molecule. Consequently, line positions calculated with an RKR potential are generally much more accurate than those obtained from Morse or other empirical potentials. In the present investigation, we have used two RKR potentials for the HF molecule. An RKR potential generated by FAlLoN 't al.22) was used in the 
initial calculations. It was found, however, that the turning points were not sufficiently dense to compute high overtone matrix elements. A new RKR potential having finer increments in $r$ was therefore generated. The matrix elements calculated with these two RKR potentials were equivalent for small $\Delta v$ transitions, but were quite different for higher overtones $(\Delta v>3)$. The line positions calculated with either RKR potential differed from measured line positions by less than fifteen wavenumbers, even at the highest vibrational and rotational states considered. In contrast, the line positions predicted by the Morse potential varied from the measured values for HF by more than two hundred wavenumbers. The preceding is not to be taken as an argument that the matrix elements calculated with the RKR potential are better than those calculated with the Morse function, since a potential which exactly reproduces the energy levels of a molccule is not unique. ${ }^{23,24)}$ Different wave functions can be derived from potentials constructed from the same set of energylevel data, and thus even if the exact form of the dipole moment were known, the computed matrix elements would not necessarily be unique. However, when two potentials give results which agree closely, it might be assumed that the molecule is being modeled reasonably well.

\subsection{Effect of potential functions on vibrational matrix elements}

Let us express the matrix elements in the form of equation (18):

$$
\left\langle l^{\prime}, J^{\prime}|\mu(r)| l, J\right\rangle=\left\langle v^{\prime}|\mu(r)| l\right\rangle \sqrt{\left[F_{v^{\prime}, v^{\prime}}(m)\right] .}
$$

The $J$-dependent $F$-factors will be discussed in a later section of this report. Our concern here is with the vibrational matrix elements, $\left\langle v^{\prime}|\mu(r)| v\right\rangle$. The effect of the potential function on the vibrational matrix elements can be seen by inspection of Figs. 1-7. Figures 1-6 compare the $\Delta t=0,1,2,3,4$ and 5 matrix elements calculated with the Morse potential function to those calculated with the RKR potential function. The wave functions have been computed numerically from equation (3) for $J=0$ and the matrix elements (equation 10) have been computed numerically with the polynomial form of $\mu(r)$ truncated after the cubic term. This approximation to $\mu(r)$ will be written $\mu(3 p)$. The dipole and Morse parameters used for all calculations are given in Table 1.

It is clear that matrix elements involving small $v$ depend very little on the potential function. However, for larger $v$, the dependence of the matrix elements on $V(r)$ becomes more pronounced. It can be concluded that as $v, v^{\prime}$ and $\Delta v$ increase, the differences in the computations for RKR and Morse potentials also begin to increase noticeably. This is to be expected, since the Morse parameters used for the computations were chosen to agree with line positions of small $\Delta v$ transitions and the RKR potential was determined using all line-position data available. The RKR potential is tabulated in Appendix I.

\subsection{Effects of potential functions on the F-factors}

A number of authors have obtained analytical expressions for the $F$-factors for the lower vibration transitions. HeRmAN, ROTHERY and RUBIN (HRR) ${ }^{(13)}$ considered the case of a rotating Morse oscillator with a linear dipole moment. A comparison of the analytically calculated $F$-factor of HRR to our Morse and RKR potential calculations for the fundamental band of HF shows that all three methods give identical results. In Fig. 8, the results of a similar calculation for the $4 \rightarrow 5$ band of $\mathrm{HF}$ have been compared with the 
TABLE 1. DIPOLI:-MOMENI OHIHITIENTS

$$
\begin{aligned}
& \text { RKR poltential } \\
& \text { (Potential parameters as in Appendix I, } r=0.91717 \times 10^{\circ} \mathrm{cm} \\
& \text { ial }(1)) \quad \mu(2 p) \quad \mu(3 p) \\
& M_{10}-1.7957 \quad H_{0}=1.7960 \quad M_{0} \quad 1.7965
\end{aligned}
$$

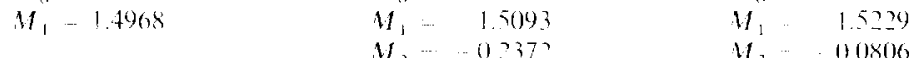

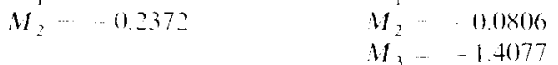

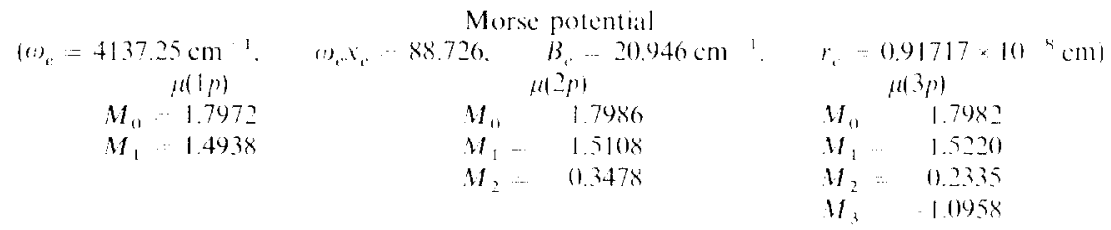

$$
\begin{aligned}
& H_{i}=\left(\begin{array}{l}
10^{x} \\
\text { min }
\end{array}\right)^{1} \text { Debye } \\
& \text { Fiperimental parameters } \\
& \text { (0) } \mu(r)(0) \quad 1.819 \times 10^{1.8} \mathrm{es}(\mathrm{I}-\mathrm{cm} \\
& 1|\mu(r)| 0>=9.850 \times 10^{20} \text { esu-cm (Ref. 21 } \\
& 3|\mu(r)| 0 \quad-1.253 \cdot 10 \quad 210 \mathrm{estl-cm}(\mathrm{Ref}, 3)
\end{aligned}
$$

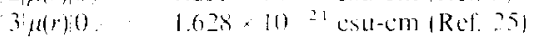

HRR calculations for the $1 \rightarrow 2$ band, the highest comparable $\Delta r=1$ transition calculated by HRR. This comparison indicates the underestimation of the fractor that is expected if HRR is used rather than the numerical computations. It can be seen that the Morse and the RKR potential give nearly identical results, whereas the HRR $F$-factor is significantly different. Figures 9 and 10 show the results of these three methods when they are applied to the first overtone $(0 \rightarrow 2)$ band and then to the second overtone $(0 \rightarrow 3)$ band of HF. All three methods give nearly the same results for the $0 \rightarrow 2$ band. Figure 10 illustrates however that the HRR approximate theory fails when applied to the second overtone band. In this latter case, the Morse and the RKR potentials also give significintly different results.
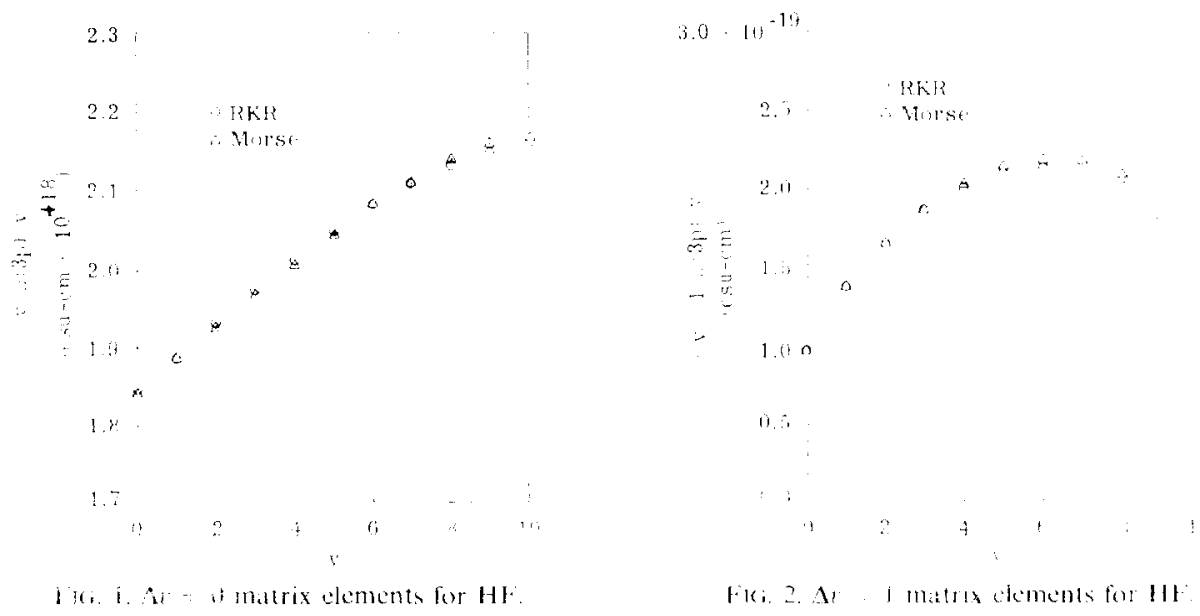


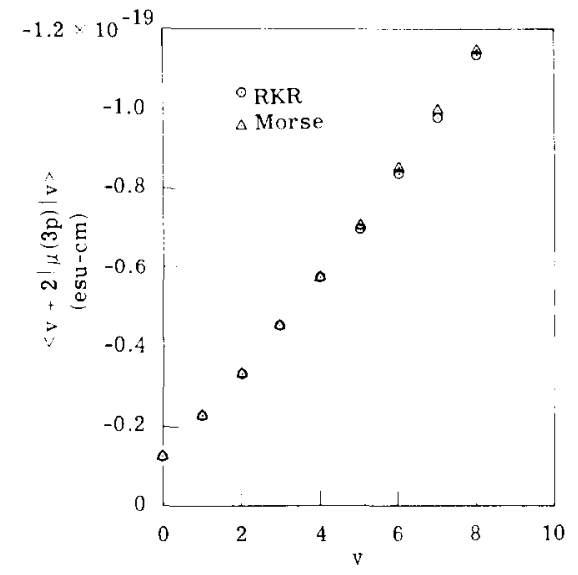

Fig. 3. $\Delta v=2$ matrix elements for HF.

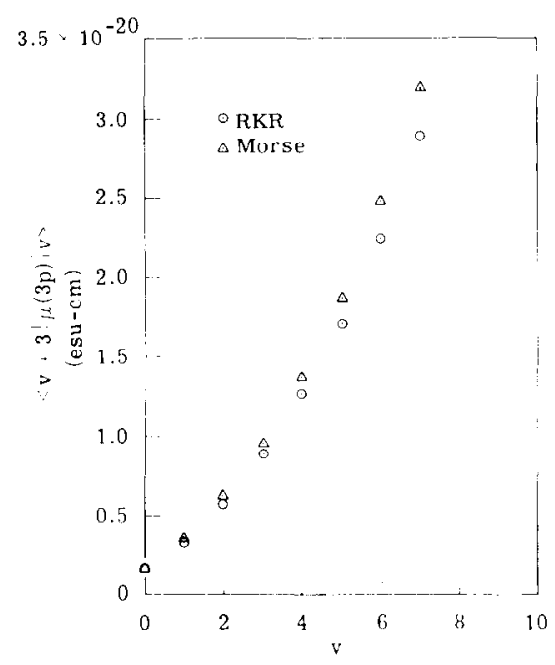

Fig. 4. $\Delta v=3$ matrix elements for HF.

\section{INFLUENCE OF THE ELECTRIC DIPOLE-MOMENT FUNCTIONS ON THE MATRIX ELEMENTS}

\subsection{Polynomial approximation}

It will be shown that the dipole moment $\mu(r)$ has a much greater influence on the matrix elements than does the mechanical description of the molecule. A comparison between two forms of $\mu(r)$ follows. The most commonly used expression for $\mu(r)$ is the truncated Taylor-series expansion about the equilibrium separation:

$$
\mu(r) \cong \sum_{i} M_{i}\left(r-r_{e}\right)^{i}
$$

The $M_{i}$ are taken as parameters to be determined from experimental measurements. Usually, as many parameters are taken as there are measurements available for that molecule, and a set of simultaneous equations are solved for the $M_{i}$ 's. Generally, the

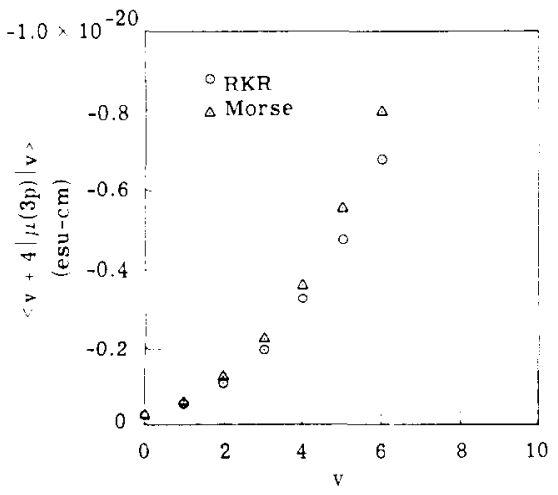

FIG. 5. $\Delta v=4$ matrix elements for HF.

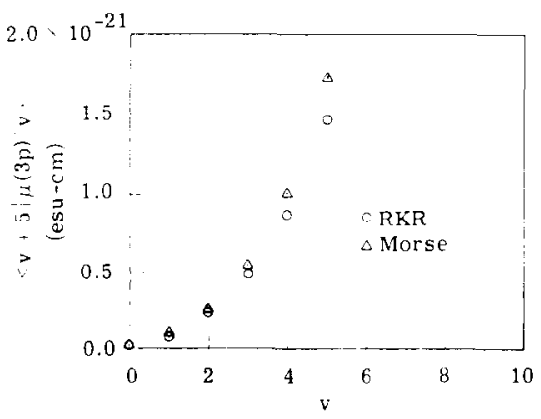

FIG. 6. $\Delta t^{\prime}=5$ matrix elements for HF. 


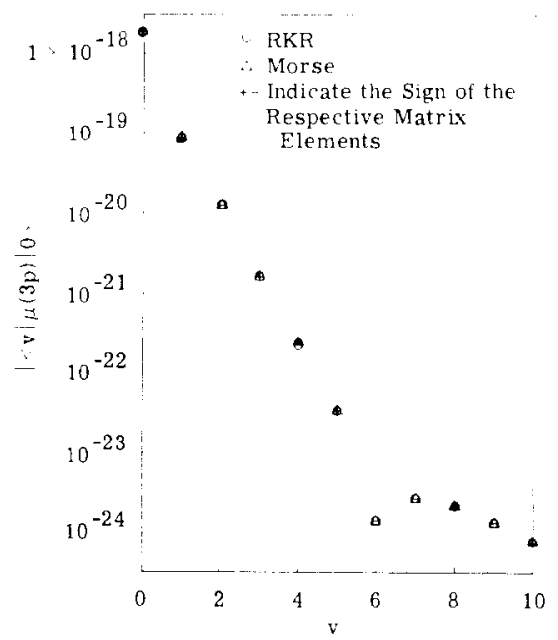

Fig. 7. Overtone matrix elements for HF connecting $!=0$.

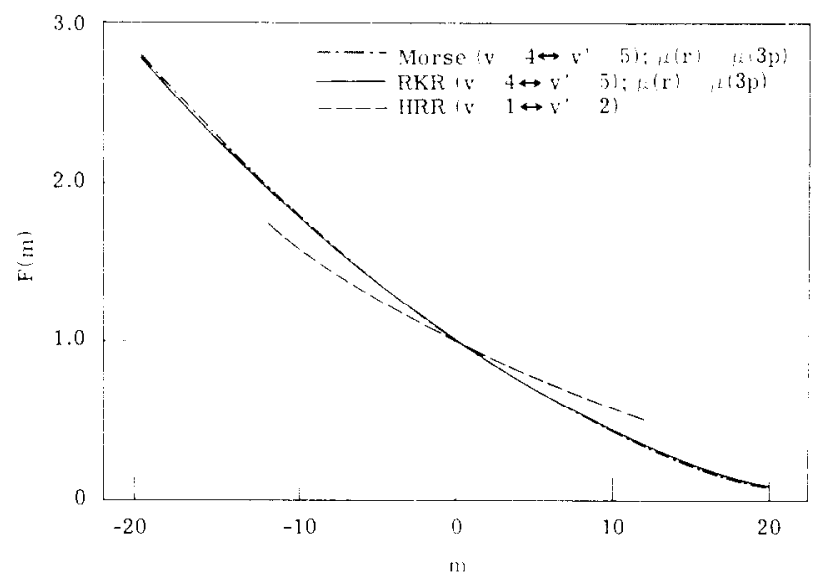

Fic. 8 . Comparison of computed HF vibration rotation interaction factors with HRR theory

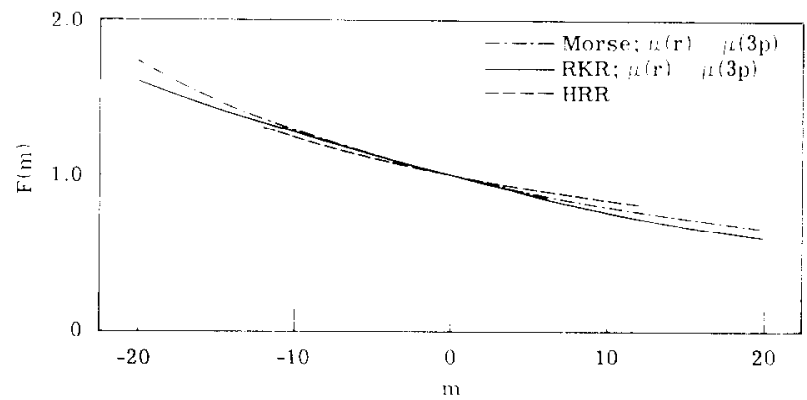

Fik. 9. Hr vibration rotation mnteracton factors for $l=1 \leftrightarrow r-2$. 


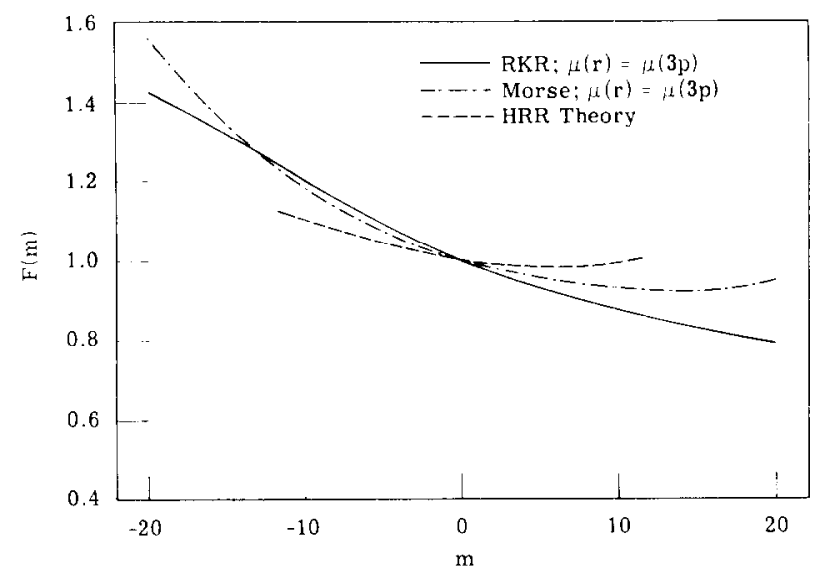

FiG. 10. HF vibration-rotation interaction factors for $t=0 \leftrightarrow t=3$.

overtone sequence measurements are the experimental information; thus, the equations are:

$$
\begin{gathered}
\langle v|\mu(r)| 0\rangle=\sum_{i=0}^{v_{\max }} M_{i} \int \psi_{v}\left(r-r_{e}\right)^{i} \psi_{0} r^{2} \mathrm{~d} r \\
\text { for } r=0 \cdots v_{\max }
\end{gathered}
$$

where $v_{\max }$ is the upper state of the highest overtone-data available. $M_{0}$ is often taken to be equal to the permanent dipole moment, and the first equation is eliminated. The remaining equations are unaffected, since because of the orthogonality of the eigenfunctions. terms containing $M_{0}$ appear only in the first equation. The rotationless matrix elements on the left-hand side of equation (2) may be determined only within an ambiguity in sign by band-intensity measurements, since the measured intensity is proportional to their square, $\langle v|\mu(r)| 0\rangle^{2}$. This ambiguity can be resolved by additional information obtained from other band measurements (BENEDICT et al. ${ }^{(1)}$ ) or through the measurement of a number of individual lines in each band (MFRFDITH ${ }^{(3)}$ ). The values of the integrals in the above equations can be determined in closed form if the harmonic, Morse, or certain other functions are used or they can be evaluated numerically, as in our present calculations. Once these values are determined, the system of $v_{\max }$ equations in the same number of unknowns may be easily solved. Generally, this procedure will determine different coefficients, $M_{i}$, for different potential functions, even for the same values of the experimentally measured matrix elements. Also, as $v_{\max }$ is increased to $t_{\max }+1$ by the inclusion of an additional measurement, all of the $M_{i}$ 's previously determined will change value as a nonzero value of $M_{v_{\max }+1}$ is determined.

The dipole-moment function as determined above can then be used as a method of interpolation and extrapolation to calculate any other matrix elements of interest :

$$
\left\langle v^{\prime}|\mu(r)| v\right\rangle \cong \int \psi_{v^{\prime}}\left[\sum_{i=0}^{v_{\max }} M_{i}\left(r-r_{e}\right)^{i}\right] \psi_{v} r^{2} \mathrm{~d} r .
$$




\subsection{Ware-function approximation}

A completely different, and more elegant approach to the analysis of experimental data was suggested in 1959 by TRISCHKA and SALwIN. ${ }^{1+1}$ In this approach, the dipole moment is expanded in terms of the radial wave function of the molecule:

$$
\mu(r) \cong \sum_{i} A_{i} \psi_{(1)}(r)
$$

When the expansion is substituted into the integral which defines the matrix element. the $A_{i}$ are determined as the matrix elements $\langle i|\mu(r)| 0\rangle$. Substituting $(30)$ into $\langle i|\mu(r)| 0\rangle$. we obtain

$$
\langle i|\mu(r)| 0\rangle=\int \psi_{i} \sum_{k=0}^{\infty} A_{k} \sum_{\psi_{0}}^{\psi_{k}} \psi_{0} r^{2} \mathrm{~d} r
$$

Since the wave functions are orthonormal, only onc term of the summation remains:

$$
\langle i|\mu(r)| 0\rangle=A_{i} \int \psi_{i} \psi_{i} r^{2} \mathrm{~d} r
$$

or

$$
A_{i}=\langle i|\mu(r)| 0\rangle \text {. }
$$

As in the polynomial expansion, one coefficient in the dipole-moment expansion is determined for each experimental measurement. In the polynomial case. the relationship between the $M_{i}$ and the measured matrix elements is somewhat obscure, since it occurs through a set of linear equations. With the wave-function expansion. the relationship is the most straightforward possible- an identity.

The substitution of the wave-function expansion for the dipole moment gives the following expression for any other transition.

$$
R^{r^{\prime \prime}, r}=\int \psi_{r} \cdot \sum R^{i,{ }^{\prime \prime}} \psi_{i} \psi_{0} \psi_{r} r^{2} \mathrm{~d} r
$$

where the notation $R^{r^{\prime},} \equiv\left\langle v^{\prime}|\mu(r)| v\right\rangle$ is used. When the summation is removed from under the integral, we have

$$
R^{r^{\prime}, r}=\sum_{i} R^{i, 0} \int \psi_{r^{\prime}} \psi_{i} \psi_{i} \psi_{r^{\prime}} r^{2} \mathrm{~d} r
$$

The sum should be over the bound states of the molecule and should include an integral (see CASHION ${ }^{(26)}$ ) to account for possible transitions to unbound states. For diatomic molecules in the ground state, transitions to unbound states are highly unlikely, so that integral contribution is assumed to be zero. In addition, the overtone matrix elements generally decrease quite rapidly as the upper state increases: therefore. Trischka and Salwen suggest that a reasonable approximation is to assume the unknown $R^{i, 0}$ be taken as zero.

In 1963. CASHION ${ }^{(26)}$ extended the work of Trischka and Salwen in an attempt to determine all matrix elements involving the $v=0$ level as a function of only one experimentally 
determined matrix element. The treatment is well documented, and therefore it will not be reviewed here. Since intensity data are now available through the second overtone band for HF, the Cashion extension has been used here only for $A_{i}$ for which $i \geq 4$.

In this paper, when the dipole-moment expressions are compared, the truncated polynomial form will be written $\mu(n p)$, where $n$ is the degree included (e.g. the linear approximation is written $\mu(1 p))$. The wave function expansion will similarly be written $\mu(n w)$, where $n$ is the number of experimental bands included.

\subsection{The $H F$ dipole inoment}

In principle, the dipole-moment function of a molecule is well defined by the electronic structure of the molecule and can be calculated without reference to band-intensity measurements. The only assumption necessary is the separability of the electronic motion from the vibrational and rotational motion, the Born-Oppenheimer approximation, which is almost always assumed in any analytic treatment. The calculation of the dipole moment requires the calculation of the electronic molecular eigenfunctions and an appropriate averaging of these eigenfunctions at a number of internuclear separations. Such a calculation for the $\mathrm{HF}$ molecule has been done by $\operatorname{NESBET}^{(27)}$ who used an approximate Hartree Fock method.

In that paper, Nesbet reports two types of calculations, a low precision calculation for three values of the internuclear separation near the equilibrium separation and a higher precision calculation for the internuclear distance approximately equal to the equilibrium distance. A comparison of the values of the dipole moment and its derivatives at the equilibrium internuclear distance obtained by Nesbet and from our measurements is given in Table 2. From that table, it can be seen that the higher precision Hartree Fock calculation gives excellent agreement with the measured dipole moment; however, since the high precision calculation was only performed for the one internuclear distance, the derivatives cannot be evaluated. Using Nesbet's lower precision calculation, we can compare the derivatives of the dipole moment which are of primary concern in determining infrared band intensities. The first derivative at the equilibrium separation is approximately 25 per cent larger than the value inferred from band-intensity measurements. ${ }^{*}$ That small a difference is quite reasonable for this type of calculation; however, it is still much larger than the approximately 3 per cent error in the value derived from the intensity measurement. The second derivative, however, does not agree with the value obtained from intensity

TABle 2. COMPaRison OF THE HF DIPOLE MOMENT AND ITS IERIVATIVES

\begin{tabular}{|c|c|c|c|c|}
\hline & & \multirow[t]{2}{*}{ Present Calculation } & \multicolumn{2}{|c|}{ ab initio Calculation $|27|$} \\
\hline & & & Calculation I & Calculation II \\
\hline 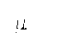 & $\mathrm{D}$ & 1.819 & 1.9614 & 1.827 \\
\hline$\frac{\mathrm{d}_{1} \mathrm{l}}{\mathrm{d} \overline{\mathrm{R}}}$ & $\mathrm{D} / \mathrm{Bohr}$ & 0.805 & 1.029 & $\mathrm{~N} A$ \\
\hline$\frac{\mathrm{d}^{2} \frac{\mu}{2}}{\mathrm{dR}^{2}}$ & $\mathrm{D} / \mathrm{Boh} \mathbf{r}^{2}$ & -0.076 & 0.260 & $\mathrm{~N} / \mathrm{A}$ \\
\hline
\end{tabular}

* In his paper, Nesbet reports somewhat better agreement between the first derivative obtained in his calculation and that obtained from band intensity measurements. It appears that that agreement was caused by a numerical error made by Nesbet. 
measurement and. in fact, differs in sign. In view of the excellent agreement obtained for the dipole moment by the high precision calculation, it is unfortunate that this type of calculation was not carried out over a range of internuclear distances.

In addition to the information about the dipole-moment function near the equilibrium separation provided by ab initio calculation, the general form of the function for large and smatl $r$ can also be determined. Nesbet concluded that at the limit of large internucleat separation, the molecule becomes two neutral atoms and in this limit. the dipole moment must approach zero. In the limit $r \rightarrow 0$. Jams a al. ${ }^{128}$ conclude that the dipole moment must approach zero as the third power of the internuclear separation. However the very strong nuclear repulsive forces prevent molecular oscillations in this region. and therefore. intensity measurements can provide litte information about this section of the dipolemoment function. In Fig. 11, $\mu 1, p), \mu(2 p)$ and $\mu(3 p)$ are shown. None of these polytromial approximations fulfill the necessary condition discussed above. However, $\mu(3 p)$ mil! represent a reasonable approximation for calculation. If it is extrapolated to larger radius. it may be seen that $\mu(3 p)$ would equal zero al approximately 4.5 Bohr radii. This is somewhat smaller than the expected zero at about 4.95 Bohr radii. which is the classical turning point for the highest observed vibrational state. ${ }^{(2)}$ For small separations. $\mu(3 p)$ clearly does not approach the origin, but. that is of little significance. since the mechanical motion is const rained by the potential to be in the region with a separation of greater than 1.15 Bohrs in even the highest observed vibrational state. Figure 12 shows the approximate dipole moment calculated when we use the permanent moment and the overtome matuis elements with the wave-function expansion. Inspection of Fig. 12 shows that this approximation does not fulfill the necessary conditions for a realistic dipole moment, since it increases exponentially at large radii. When we add more terms to the wave-function expansion and use Cashion's values for the overtone matrix elements (lig. 13) we halve a much more realistic dipole-moment curve over a wider range of internuclear distance: but there is still a rapid and unreasonable increase at large $\%$ probably accounting for the wery large $\Delta r=1$ matrix elements calculated for higher vibrational transitions when this type of expansion is used (Section 3.4). In fact, both the watse-function and the polynomial expansions are constrained by the forms of their respective functions to approath positive of negative infinity for large $r$. The exponential increase characteristic of the wave-function expansion generally dominates the dipole-moment function at a smaller radius than does the increase of the highest degree term retaned in the polynomial expansion. Therefore. the wave-function expansion seems to extrapolate less reasonably to intermediate radii. This probably accounts for the erratic behavior of the matrix elements as a lunction of i with the wave-function approximation fealculated in Section 3.4).

\subsection{Dependence of the rotationkess matrix tements on the dipole-moment finction}

Precise definition of the dipole-moment function is the most important ingredient in the calculation of matrix elements. The significance of this function is illustrated by Fig. 1t. which compares $\mu(2 p)$ - and $\mu(3 p)$-overtone matrix elements for the RKR potential. Significantly. differences occur. though $\mu(r)$ is nearly identical near $r=r_{\text {c }}$ and diverges only near the turning points (see Figs. 11 1.3). The RKR calculations made with the thirddegree polynomial dipole moment can be compared with similar calculations made with the three-coefficient wave-function expansion of Trischka and Salwen and with Cashion's 


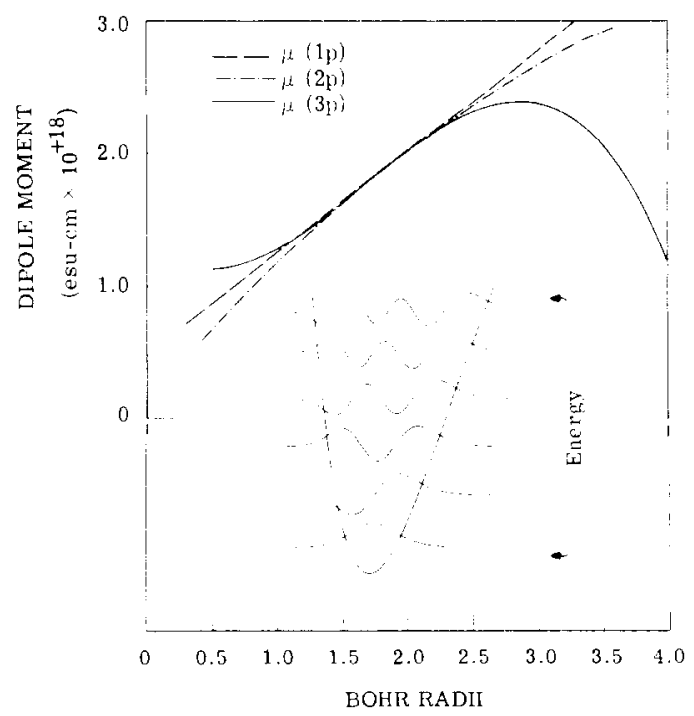

FIG. 11. HF dipole moments when the RKR potential is used.

theory (Figs. 15-17). As Figs. 15 and 16 show, for the $\Delta v=1$ and $\Delta v=2$ calculations, the polynomial and the wave-function expansion give very similar results for the lower vibrational transitions; however, the results diverge quite quickly for higher vibrational transitions and in the case of $\langle 9|\mu(r)| 10\rangle$ matrix element, differ by an order of magnitude. The rapid increase of these matrix elements calculated with the wave-function approximations seems unreasonable at large $v$. Some explanation for this unexpected behavior was

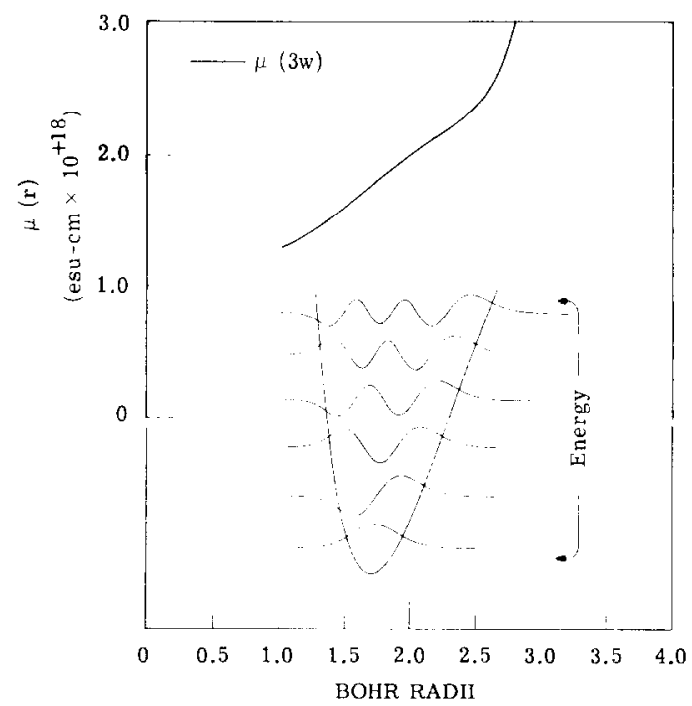

FIG. 12. $\mu(3 w)$ for HF when the RKR potential is used. 


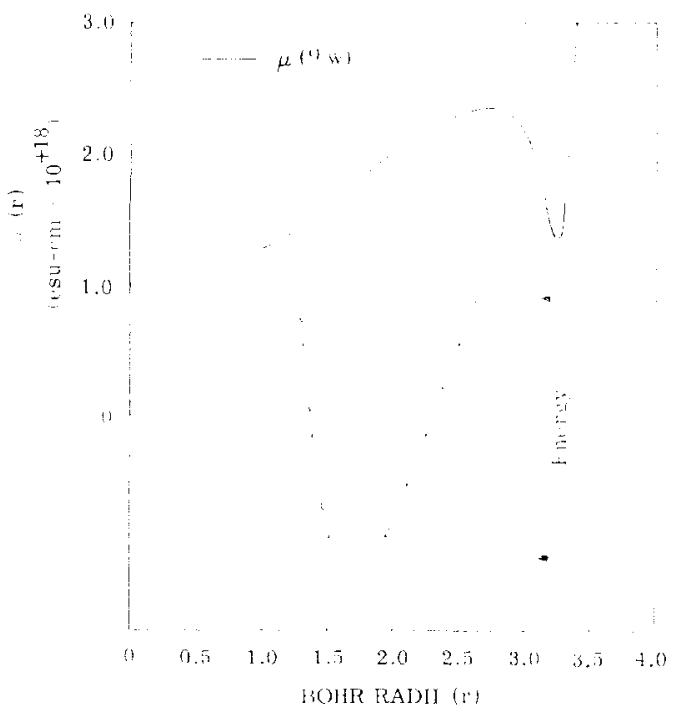

Fia. 13. M $^{9}$ for HF when the RKR potential is used (matrix elements determined from Cashion: extrapolation for $i$. 3i.

suggested in Section 3.3. In Fig. 17, the overtone matrix elements predicted with the use of the $\mu(3 p)$ dipole moment can be compared with those arrived at by Cashion. The signs in the respective symbols represent the signs of the matrix elements represented. It can be seen from Fig. 17 that a correspondence between these two methods seems to hold through the fourth overtone $\langle 5|\mu(r)| 0\rangle$ matrix element. For large overtone matrix elements, differences in both sign and magnitude are considerable. The corresponding overtone elements from Trischka and Salwen's wave-function approximation are the same as the first three overtone elements in the third-degree polynomial approximation represented in Fig. 17. sinee both approximations are fit to experimental data : the higher overtone matrix elements for the wave-function expansion are. by definition, zero and therefore are not plotted. A complete tabulation of the rotationless matrix elements and Einstein coefficients calculated with the $\mu(3 p)$ approximation are tabulated in Appendix 2.

\subsection{Rotational dependence of the matrix elements when the polyomial dipole moment is used}

The $F$-factors which represent the rotational dependence of the matrix elements are also affected by the choice of the dipole-moment function. Figures 18.22 show what cffect the addition of the third-degree term to the polynomial dipole-moment function has on the HF $F$-factors. The fundamental band $F$-factor has not been included. since in this case, both polynomial functions give identical results which agree quite well with experiment (sec Ref. 2). Figure 18 compares the calculated and measured $F$-factors in the first overtone. For $m>0$ corresponding to R-branch transitions, the third-degree polynomial calculation gives slightly better results; for $m<0$, the second-degree polynomial calculation agrees more closely with the measured values. However, definite conclusions cannot be drawn from the comparison because of error in the measurement. Figure 19 will give an additional 


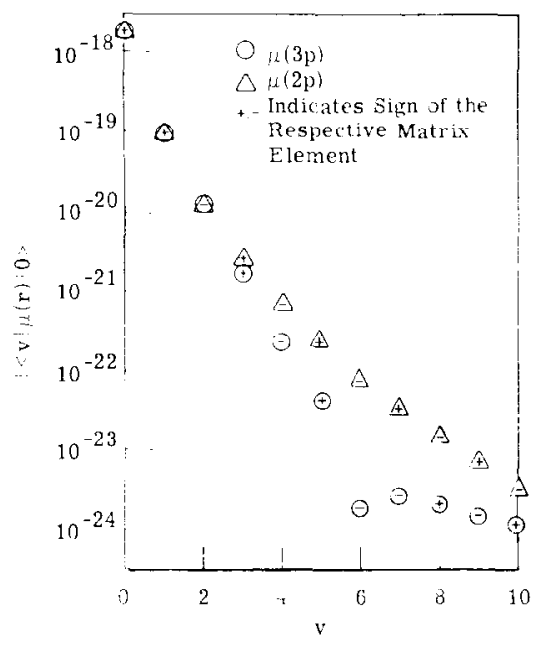

FIG. 14. Overtone matrix elements for HF when various dipole moments are used with the RKR potential.

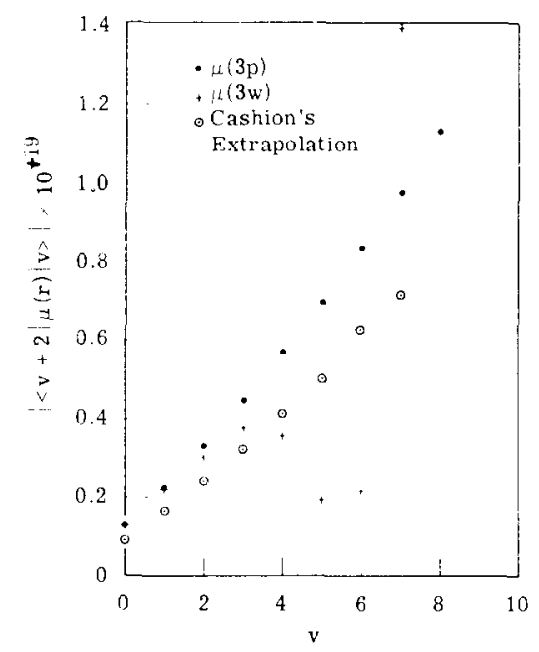

FIG. 16. $\Delta v=2$ matrix elements for $\mathrm{HF}$ when the RKR potential is used.

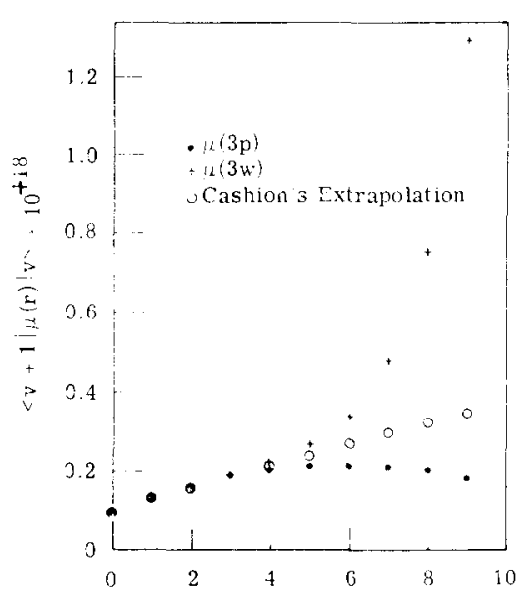

Fig. 15. $\Delta t=1$ matrix elements for $\mathrm{HF}$ when the RKR potential is used.

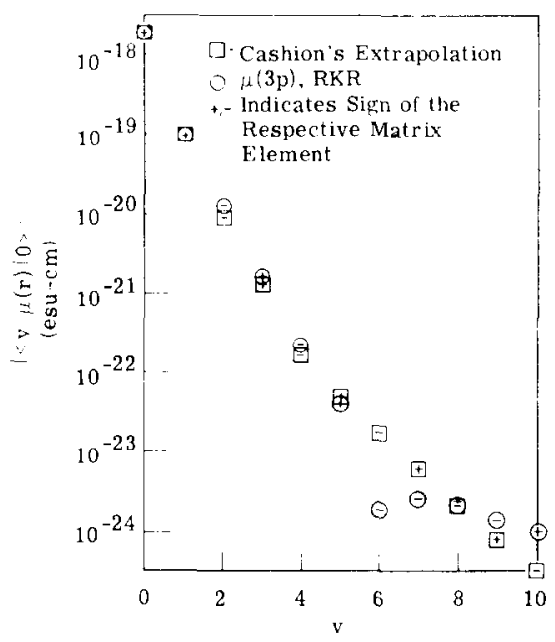

FIG. 17. Overtone matrix elements for $\mathrm{HF}$ when the RKR potential is used.

comparison of the $F$-factors when the $0 \rightarrow 3$, large $J$ line strength measurement (now in progress) is completed. Figures 20 and 21 show two $\Delta v=1 F$-factors calculated with the different polynomial dipole moment for high vibrational transitions. Significantly, the additional term does have some effect, although approximate analytic theories ${ }^{(10,13)}$ predict that the $F$-factors for $\Delta v=1$ transitions should be dependent only on the coefficient 


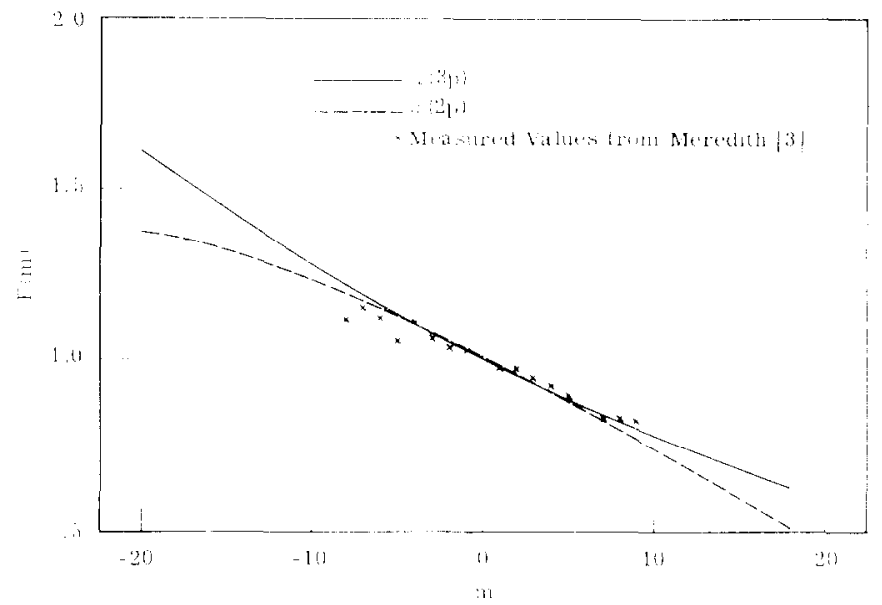

Fic. 18. Vibration rotation interaction for the $t=0+\cdots, t=2$ band of $\mathrm{HF}$ when $\mathrm{RKR}$ potential is used.

of the linear dipole-moment approximation term. Clearly, those theories do not hold for higher $l$ and $J$ transitions, as shown in Fig. 22, a comparison of Meredith s extension of the Herman Wallis theory with the $F$-factor calculated numerically for the $3 \rightarrow 4$ band.

\section{ERROR ANALYSIS FOR THE VIBRATIONAL MATRIX ELEMENTS}

In addition to the minor errors incurred by inaccuracies of the potential function and by numerical error in the calculation, there are two other sources of error in the present calculations. These two remaining sources of error will be designated approximation error and measurement-induced error. By approximation error, we mean the error introduced into the calculated matrix elements because the form of the chosen dipole-moment

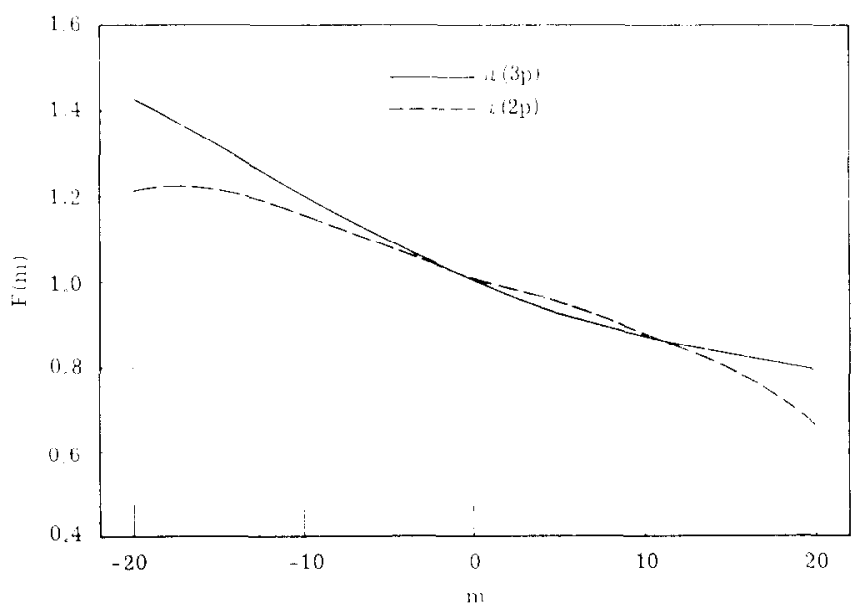

Fig. 19. Vibration rotation interaction for the $v=0 \leftrightarrow v=3$ band of HF when RKR potential is used. 


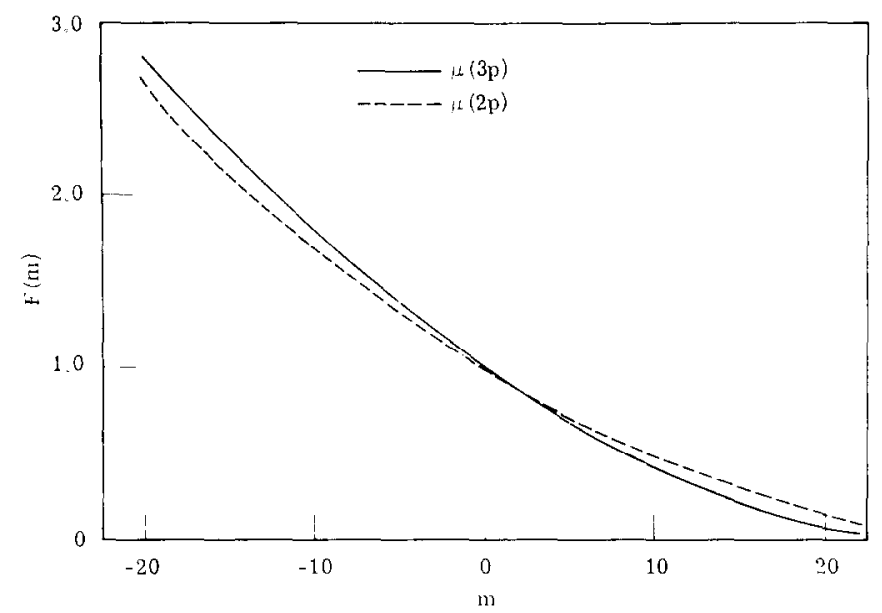

FIG. 20. Vibration-rotation interaction factor for the $t=4 \leftrightarrow v==5$ band of HF when the RKR potential is used.

approximation does not correctly represent the real dipole moment of the molecule. For example, in the case of the polynomial approximation, we are assured that if enough terms are retained, we can adequately represent any reasonable dipole-moment function. However, lack of experimental overtone information limits the number of terms which can be added to the polynomial approximation. Moreover, it is not possible to check the reliability of the approximate solution, since little is known about the actual form of the dipole moment.

The measurement-induced error present in the calculated matrix elements is easier to handle. We define measurement-induced error as error in the calculated matrix elements

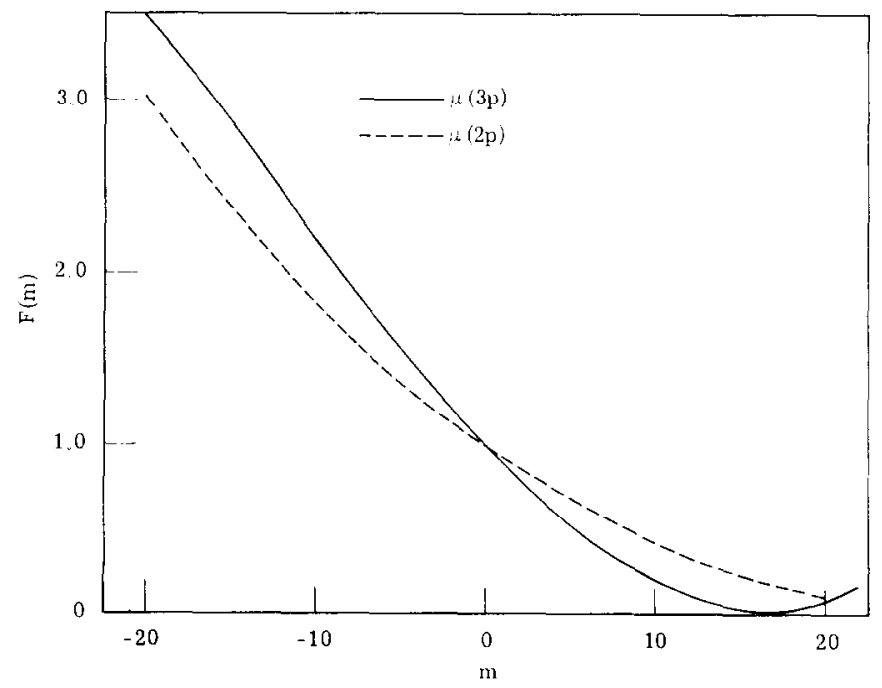

FIG. 21. Vibration-rotation interaction factor for the $v=8 \leftrightarrow v=9$ band of HF when the RKR potential is used. 


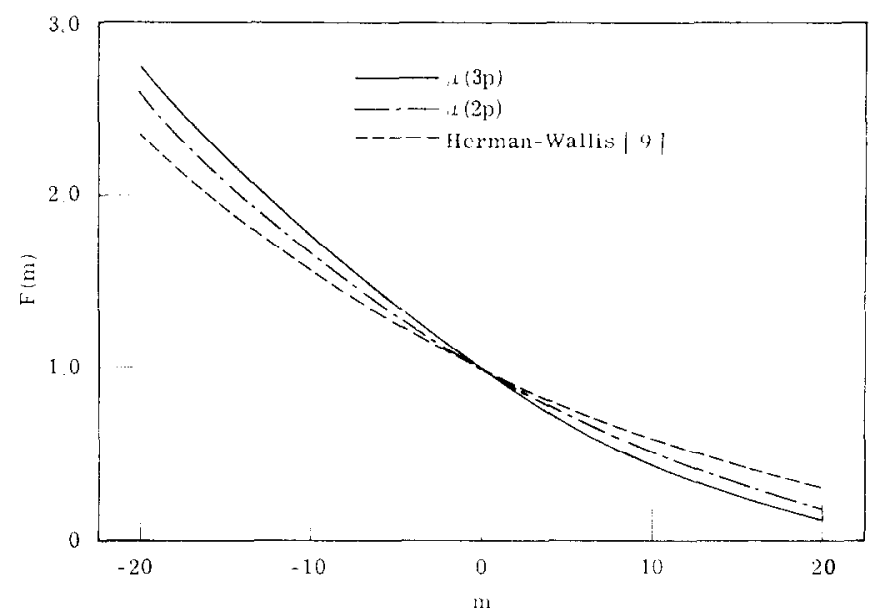

Fis. 22. Vibration rotation interaction factor for the $t=3 \leftrightarrow v=4$ band of $H F$ when the RKR potential is used.

caused by inaccurate measurements of the overtone matrix elements used to determine the dipole moment coefficients when the chosen dipole-moment approximation is adequate. For example, consider the third-degree polynomial approximation. If we assume that the dipole moment of the molecule is well represented by a third-degree polynomial, then we could find the correct polynomial by using the uniqueness theorem for polynomials, by solving the set equations in Section 3.1 using the correct matrix elements through $\langle 3|\mu(r)| 0\rangle$. However, for the overtone matrix elements, we must use measured values which may contain some errors; therefore, generally, the coefficients of $\mu(3 p)$ will be in error. In turn. these errors introduce other errors into matrix elements calculated with that particular polynomial dipole-moment approximation. A representation of the magnitude of those induced errors is the aim of this section.

\subsection{Deritation of the measurement-induced error expression}

For clarity, matrix notation will be used in the derivation of the measurement-induced error expression. For $\mu(n p)$, the coefficient $M_{i}$ are solutions of the following set of $n+1$ equations:

$$
\begin{aligned}
& \sum_{i=-1)}^{n} M_{i} \int \psi_{0} \rho^{i} \psi_{0} r^{2} \mathrm{~d} r=\langle 0|\mu(r)| 0\rangle \\
& \sum_{i=1}^{n} M_{i} \int \psi_{0} \rho^{i} \psi_{\imath} r^{2} \mathrm{~d} r=\langle l|\mu(r)| 0\rangle \quad \text { for } r=1,2 \ldots n
\end{aligned}
$$

where

$$
\rho \equiv \frac{r-r_{e}}{r_{i}}
$$

Since we are not presently interested in the pure rotation transitions and $M_{0}$ appears only in the first equation of (35), we may restrict our attention to the last $n$ equations of (35). 
Those equations may be written in matrix notation as:

$$
A M=R^{0}
$$

where $A$ is a matrix which has elements, $A_{v i}$ :

$$
A_{v i}=\int \psi_{0} \rho^{i} \psi_{v} r^{2} \mathrm{~d} r
$$

where $M$ is a $1 \times n$ matrix which has elements, $M_{i}$. The elements of $R^{0}$ are the measured overtone matrix elements

$$
R_{i}^{0}=\langle i|\mu(r)| 0\rangle
$$

If $A^{-1}$ exists, $M$ may be found:

$$
M=A{ }^{1} R^{0}
$$

Any matrix elements can be calculated for $\mu(n p)$ :

$$
\langle k+\Delta v|\mu(r)| k\rangle=\sum_{j=1}^{n} M_{j} \int \psi_{k} \rho^{j} \psi_{k+\Delta v} r^{2} \mathrm{~d} r .
$$

If the following definitions are assumed for the matrices $B^{\Delta v}$ and $R^{\Delta v}$

$$
\begin{aligned}
B_{k j}^{\Delta v} & \equiv \int \psi_{k} \rho^{j} \psi_{k+\Delta v} r^{2} \mathrm{~d} r \\
R_{k}^{\Delta v} & \equiv\langle k+\Delta v|\mu(r)| k\rangle
\end{aligned}
$$

then equation (36) can be written :

$$
R^{\Delta v}=B^{\Delta v} M
$$

If we substitute for the $M$ above:

$$
R^{\Delta v}=B^{\Delta v} A^{-1} R^{0}
$$

Equation (38) is particularly important because it gives the matrix element desired as a linear combination of the input matrix elements, as can be seen if equation (38) is written explicitly in terms of the elements of the matrices:

$$
\begin{aligned}
\langle k+\Delta v|\mu(r)| k\rangle & =\left[\sum_{v=1}^{n}\left(\sum_{j} B_{k j}^{\Delta v} A_{j v}^{-1}\right)\langle v|\mu(r)| 0\rangle\right] \\
& =\sum_{v=1}^{n}\langle v|\mu(r)| 0\rangle\left(\sum_{j} B_{k j}^{\Delta v} A_{j v}^{-1}\right) .
\end{aligned}
$$

The linearity of (39), coupled with the assumption of the independence of the measurements, allows us to write the variance of the computed matrix elements, $\sigma_{k, k+\Delta v}^{2}$, in terms of the variances of the overtone measurements $\sigma_{0, v}^{2}$ :

$$
\sigma_{k, k+\Delta v}^{2}=\sum_{v=1}^{n} \sigma_{0, \nu}^{2}\left(\sum_{j} B_{k j}^{\Delta v} A_{j v}^{-1}\right)
$$


Or. as standard deviations:

$$
\sigma_{k, k+\lambda}=\sqrt{\left[\sum_{i=1}^{n} \sigma_{0, r}^{2}\left(\sum_{j} B_{k j}^{v r} A_{j r}{ }^{1}\right)\right] .}
$$

The above provides the desired result, a relationship between the measurement error and the measurement-induced error in the calculated matrix elements.

\subsection{Results for calculated $\Delta t=1$ matrix elements}

Equation (40) provided a general relation for the measurement-induced error for any calculated matrix element, but here we consider only $\Delta t=1$ transitions. The clements of the matrices $A$ and $B^{\Delta:-1}$ have been calculated numerically and are given in Tables 3 and 4 for the case of $n=3$, when the RKR potential function is used. The matrix $C^{n=1}$. detined by

$$
C^{x r-1} \equiv B^{x+1} A^{1}
$$

for $n=3$. is given in Table 5. For calculation purposes, we have taken the standard error for the overtone bands measured as 3 per cent. This 3 per cent corresponds to approximately 6 per cent error in the values of the measured quantities, the line strengths. We chose 3 per cent to represent an upper bound on the probable crror. For comparison. we did a least-square fit of the measured $r=0 \rightarrow 2$ overtone strengths ${ }^{(3)}$ to a second-degree polynomial. A standard deviation of less than 2 per cent was obtained. That value corresponds to a standard error of less than 1 per cent in the $r-0 \rightarrow 2$ matrix element; however. systematic error may remain undetected.

TABIL 3. THI COLHICHENI MAIRIX. A. (OMPUTII) WHEN THF RKR POTENTIAI IS ISEL)

$\begin{array}{rrr}7.31450 \mathrm{E}-2 & 7.14120 \mathrm{~F}-3 & 1.73680 \mathrm{~F}-3 \\ -8.21549 \mathrm{E}-3 & 6.6637 .1 \mathrm{~F}-3 & 1.15390 \mathrm{~F}-3 \\ 1.63503 \mathrm{E}-3 & -1.61860 \mathrm{H}-3 & 6.00283 \mathrm{~F}-4\end{array}$

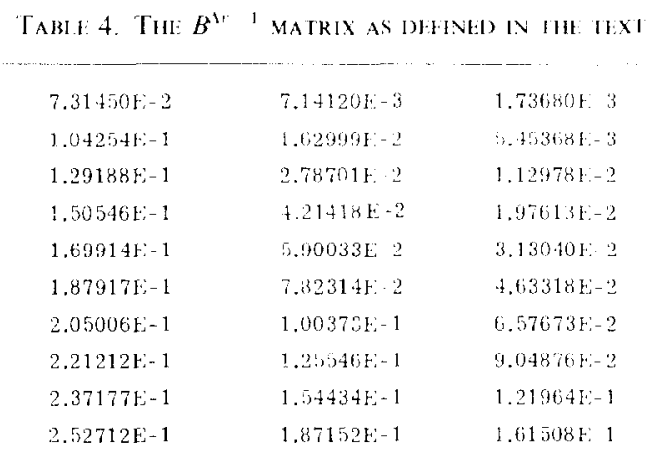


Table 5. The $C^{A \prime=1}$ Matrix which Contains the COEFFICIENTS WHICH RELATE THE MEASURED OVERTONE MATRIX ELEMENTS TO THE DESIRED $\Delta t=1$ MATRIX ELEMENT

\begin{tabular}{lll}
$1.0000 \mathrm{E} 0$ & 0.0 & 0.0 \\
$1.5325 \mathrm{E} 0$ & $1.3598 \mathrm{E} 0$ & $2.0373 \mathrm{E} 0$ \\
$2.0398 \mathrm{E} 0$ & $3.6219 \mathrm{E} 0$ & $5.9566 \mathrm{E} 0$ \\
$2.5616 \mathrm{E} 0$ & $6.9137 \mathrm{E} 0$ & $1.2219 \mathrm{E} 1$ \\
$3.1191 \mathrm{E} 0$ & $1.1335 \mathrm{E} 1$ & $2.1336 \mathrm{E} 1$ \\
$3.7203 \mathrm{E} 0$ & $1.6975 \mathrm{E} 1$ & $3.3790 \mathrm{E} 1$ \\
$4.3856 \mathrm{E} 0$ & $2.4137 \mathrm{E} 1$ & $5.0473 \mathrm{E} 1$ \\
$5.1238 \mathrm{E} 0$ & $3.3085 \mathrm{E} 1$ & $7.2318 \mathrm{E} 1$ \\
$5.9641 \mathrm{E} 0$ & $4.4289 \mathrm{E} 1$ & $1.0079 \mathrm{E} 2$ \\
$6.9163 \mathrm{E} 0$ & $5.8139 \mathrm{E} 1$ & $1.3728 \mathrm{E} 2$ \\
\hline
\end{tabular}

Using the assumed 3 per cent standard error, we have computed the $\Delta v=1$ matrix elements and the standard deviation of each and plotted these values in Figs. 23 and 24. We calculated Fig. 23 using $\mu(3 p)$ with the RKR potential function. Figure 24 represents the $\Delta v=1$ matrix elements, which we calculated using a fourth-degree polynomial expansion with the value of the third overtone $(v=0 \rightarrow 4)$ matrix element taken from Cashion's treatment. The wider error bars on that curve represent the present situation, where we have assumed the standard error associated with the $v=0 \rightarrow 4$ matrix element to be 50 per cent. The narrower error bars on that curve were calculated under the assumption that the $v=0 \rightarrow 4$ matrix element was known to 3 per cent. Thus, the narrower error bars show the improvement in our knowledge of the $\Delta v=1$ matrix elements which might be obtained if we make a measurement of the $\langle 0|\mu(r)| 4\rangle$ matrix element assuming that the fourthdegree polynomial adequately represents the dipole-moment function of the HF molecule.

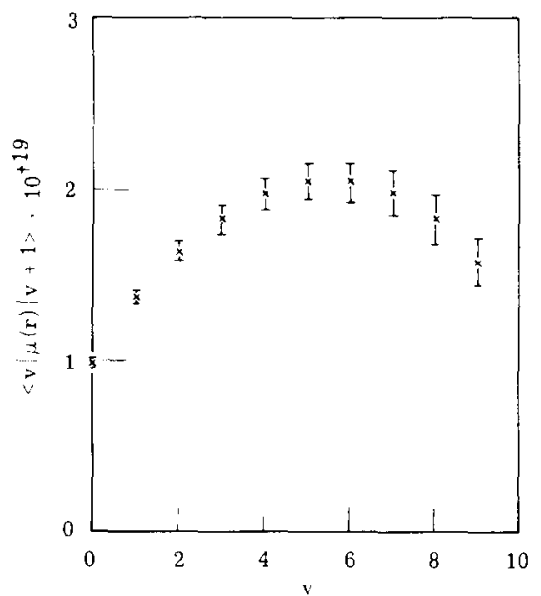

Fig. 23. Measurement-induced error in $\Delta v=1$ matrix elements when $\mu(3 p)$ is used.

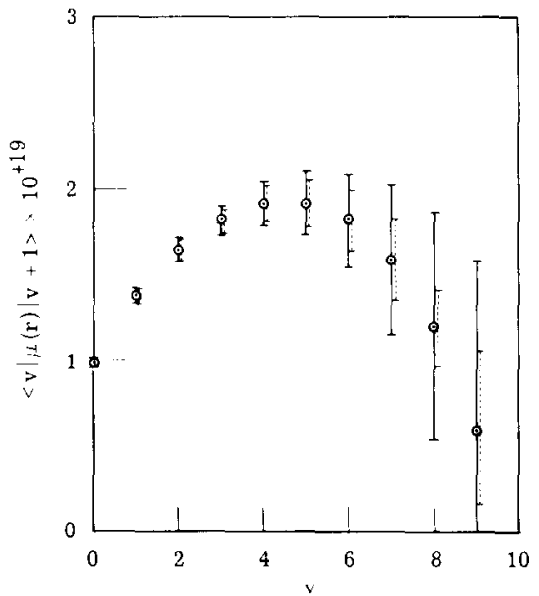

FiG. 24. Measurement-induced error in $\Delta v=1$ matrix elements when $\mu(4 p)$ is used. Error bounds are explained in text. 


$$
5 \operatorname{CONCI,LSION}
$$

This investigation has shown that the nost important factor is the calculation of vibration rotation matrix elements in the dipole moment approximation used. The influence of the mechanical model as defined by the use of a Morse or RKR potential function is much less significant and effects only transitions from the higher vibrational and rotational quantum states. Since at present ab initio theories are inadequate for accurate determinations of dipole moment parameters, the calculation of matrix elements for intermediate and large vibrational and rotational transitions is heavily dependent on the number of infrared intensity measurements available for a molecule. The primary conclusion infers that analytic theories which retain only linear or quadratic terms in the dipole moment approximation are not adequate for higher vibrational and rotational matrix element calculations. This conclusion was also explicitly confirmed in the paper. The explicit calculations also suggest that the truncated wave-function approximation to $\mu(r)$ is not appropriate for matrix element calculations involving large or intermediate vibrational states.

It is not possible to determine the absolute error in most calculated matrix elements since little experimental data is available. However, an expression has been derived for the determination of error induced into the calculated matrix elements by the experimental errors in the measured band intensities.

A complete tabulation of the numerically calculated vibration rotation matrix elements using the $\mu(3 p)$ dipole moment approximation for Hydrogen Fluoride has recently been published. (3) The alculations include $\Delta r$ 's through 5 for $r$ 's less than 10 and $J$ values to 35.

\section{APPENDIXI.}

RKR POTENIIAI. FOR $\gamma^{1} \Sigma$ ' STATH OH HYDROGHN FLIORIDE: $†$

Potential Function

The tabulated RKR potential which follows was used for the calculation, but with additional points at intermediate $r$-values to provide a more accurate function

\begin{tabular}{|c|c|c|c|}
\hline$v$ & $\begin{array}{l}\text { Fne }{ }^{2} y \\
\mathrm{irm}^{-1}\end{array}$ & $\begin{array}{l}m m \\
\mathrm{iA}^{\prime}\end{array}$ & $\begin{array}{l}r_{\max } \\
(A)\end{array}$ \\
\hline 0 & 2047049 & 1) $834345 f$ & 10203310 \\
\hline 1 & 6008.695 & 0.7346222 & 1.1129460 \\
\hline 2 & 9798.297 & $0.754925 f$ & 1.1867330 \\
\hline 3 & 13420.380 & 1). 7332200 & 1.2534800 \\
\hline 4 & 16878.720 & 0.7160859 & 1.3179500 \\
\hline 5 & 20176.520 & 0.7019794 & 1.3806320 \\
\hline 6 & 23316.520 & 0.6900074 & 1.4430680 \\
\hline$i$ & 26301.030 & 0.6797299 & 1.5057470 \\
\hline 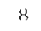 & 29131830 & 0.6707530 & 1.5693750 \\
\hline 4 & 31809.960 & 0.6628389 & 1.6345320 \\
\hline 10 & 34335.430 & 0.6558151 & 1.7018600 \\
\hline 11 & 36706.820 & 0.6495619 & 1.7721520 \\
\hline 12 & 38920190 & $0.643986 i$ & 1.8465430 \\
\hline 13 & 40971.450 & 0.6390724 & 1.9263947 \\
\hline 1.7 & 42849.960 & 0.6347828 & 2.0140000 \\
\hline $1 i$ & 44542.330 & 0.6311092 & 2.1128150 \\
\hline 16 & 46031.260 & 0.6280546 & 2.2284110 \\
\hline 17 & 47292.370 & $0.625517 \mathrm{H}$ & 2.3712500 \\
\hline 18 & 48294.480 & 0.6232452 & 2.5624320 \\
\hline 19 & 48998.130 & 0.6201019 & 28587490 \\
\hline
\end{tabular}

+ This potential was computed with a program written by ZARL:. ${ }^{211}$ Spectroscopic constants determined by JoHNS and BARROW ${ }^{(30)}$ were used as inpul data. 
Computation of electric dipole matrix elements for hydrogen fluoride

$\underline{\simeq} \underline{\Omega}$

$\delta$

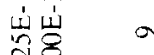

๙

s

山山山்

等导的

으유요

800

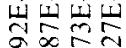

$g \stackrel{2}{2} \widehat{x}$

넹피용

$8=$

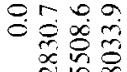

엉후

으유ํำ

山่山岀山岀

券壬寻余方

तनिक्षण

山岁䋇

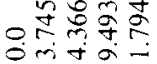

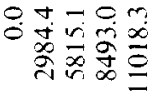

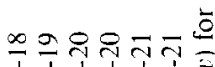

엉요

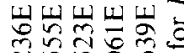

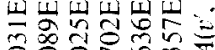

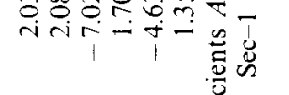

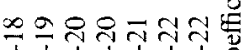

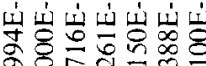

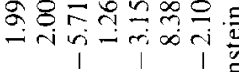

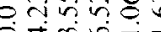

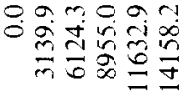

ปช่

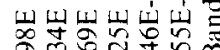

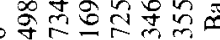

过宁守的位?

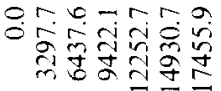

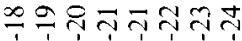

영ㅇㅇㅇㅇㅇ

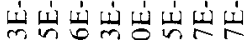

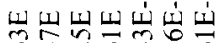

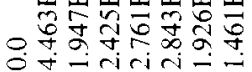

$m$

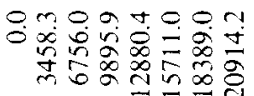

a

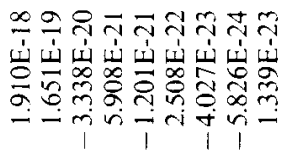

웡ㅇㅇㅇㅇㅇㅇㅇ

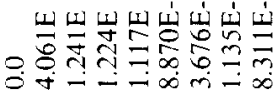

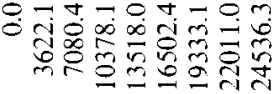

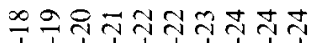

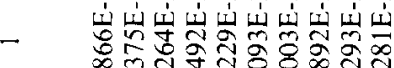

- iriog-

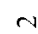

N

ปठ8:

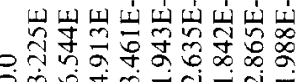

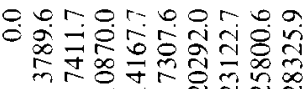

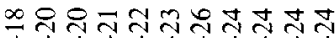

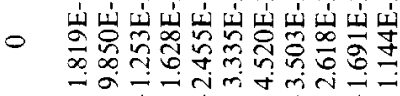

등ㅇㅇㅇㅇㅇㅇํㅇㅇำ

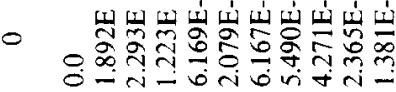

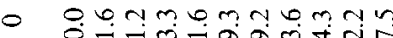

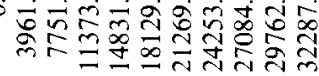

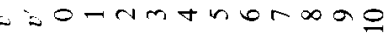

$\therefore$ a

$\therefore=0-\pi m+n$ and 


\section{REFERENCES}

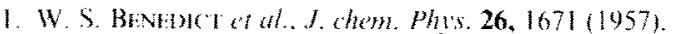

2. R. J. Lovme and W. F. Herent. J. Opt. Smo Am. 52, 1374 (1962).

3. R. E. MEREDITU, JQSRT 12,485(1972).

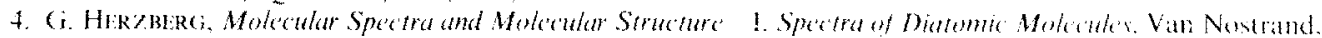
New York (1950)

5. M. BORN and J. R. OPPFNIIEIMER, Amn. Phes, 84, 457 (1927)

6. A. R. EDMoNDs, Anguhar Momentum in Questum Vechanics, Princeton ( niversity Press

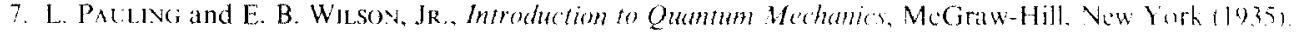

8. I. R. Opprinimik Proc. Cimb. Phil. Soc, 23, 327 (1926).

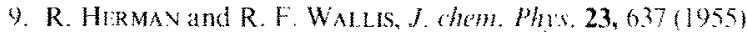

10. H. S. HFass and G. HiRZBERG. 7. Phys, 133, 48 (1952).

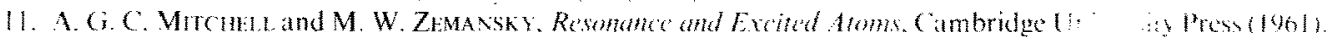

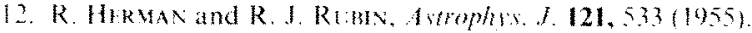

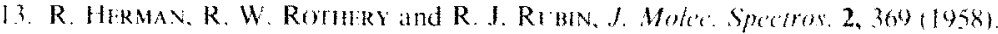

14. J. TRISCHKA and H. SALWTX, J. hem. Phis, 31, 21K (1959).

15. J. W. ('(x)I:Y. Math. (omp. 15,363(1961).

16. J. K. CASHION, J. chom. Phis. 39, $1872(1963)$.

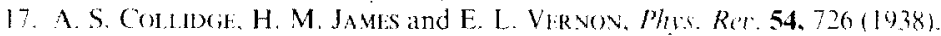

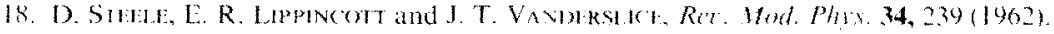

19. R. RYUBFR ( 2. Plbs, 73, 376 (1931).

20. O. KIIIN, Z.. Plun. 76, 226(19,32).

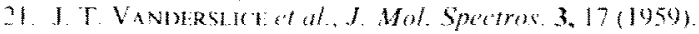

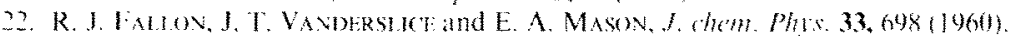

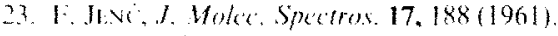

24. R. fose and W, Kolin, Phys. Rer. 88, 382 (1952).

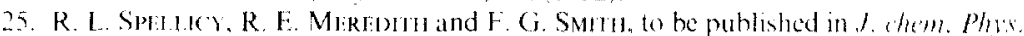

26. J. K. CAsmion. J Molec Spectros. 10, $182(1963)$.

27. R. K. NisheT. J. chem. Phix. 36. $1518(1962)$.

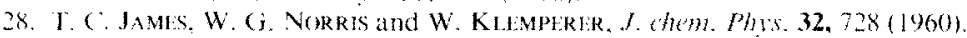

29. R. V. ZARt, Report No. UCRL-10925. University of California, Vovember (1963).

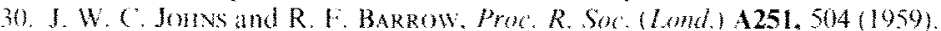

3. R. E. Mmommand I. G. Smm, Report No. 8413-39-T(11). Willow Run Laboratories of the Intitute af Seince and Technology, The University of Michigun. Ann Arbor, August 11971). 